\title{
FOLIATIONS WITH NONORIENTABLE LEAVES
}

\author{
W. G. DWYER, D. B. ELLIS AND R. H. SZCZARBA
}

\begin{abstract}
We prove that a codimension one foliation of an orientable paracompact manifold has at most a countable number of nonorientable leaves. We also give an example of a codimension one foliation of a compact orientable manifold with an infinite number of nonorientable leaves.
\end{abstract}

Introduction. The purpose of this paper is to investigate the occurrence of nonorientable leaves in a codimension one foliation of an orientable manifold. Suppose that $\mathscr{F}$ is a codimension one foliation of the orientable manifold $M$ defined by the subbundle $E$ of the tangent bundle $T M$ of $M$. Let $Q=T M / E$ be the normal bundle of $\mathscr{F}$. If $Q$ is orientable, then so is $E$ (since $T M$ is orientable) and each leaf $L$ of $F$ must be orientable (since $T L=E \mid L$ ). If $Q$ is nonorientable, then so is $E$ and the leaves of $\mathscr{F}$ may or may not be orientable. ${ }^{l}$ For example, let $M$ be the total space of the flat $S^{1}$-bundle over $R P_{2}$ defined by the homomorphism

$$
\varphi: \pi_{1}\left(R P_{2}\right) \rightarrow \operatorname{Diff}\left(S^{1}\right)
$$

with $\varphi(u)$ complex conjugation on $S^{1} \subset C, u$ nonzero in $\pi_{1}\left(R P_{2}\right)$. Then $M$ is covered by $S^{2} \times S^{1}$ and the images of the sets $S^{2} \times\{y\}, y \in S^{1}$, define a codimension one foliation of $M$. This foliation has exactly two nonorientable leaves which correspond to the two fixed points of $\varphi(u)$ (see $\S 1$ ).

On the other hand, it is not possible for all leaves of $\mathscr{F}$ to be nonorientable. In fact, a nonorientable leaf of a foliation of an orientable manifold is easily seen to have nontrivial leaf holonomy. It then follows from a result of Epstein, Millett, and Tischler [1] that the union of all nonorientable leaves is contained in the complement of a dense $G_{\delta}$.

Our result is the following.

THEOREM 1. Let $\mathcal{F}$ be a codimension one foliation of a connected orientable paracompact manifold $M$. Then $\mathcal{F}$ has at most a countable number of nonorientable leaves.

The proof of this result is given in $\S 3$. An example is given in $\S 1$ to show that the number of nonorientable leaves can be infinite.

Received by the editors February 3, 1983.

1980 Mathematics Subject Classification. Primary 57D30; Secondary 57D99.

'In a conversation with several of the authors, W. S. Massey asked whether an orientable manifold could have a foliation with nonorientable leaves. It was his question that motivated this paper. We are also indebted to Larry Conlon for helpful conversations on this matter.

Q1983 American Mathematical Society $0002-9939 / 83 \$ 1.00+\$ .25$ per page 
REMARKS. 1. If $q>1$, foliations of codimension $q$ can be constructed with an uncountable number of nonorientable leaves. For example, the flat $S^{4}$-bundle over $R P_{2}$ defined by reflection in an equator $S^{q-1}$ of $S^{q}$ has one nonorientable leaf for each pont of the equator $S^{q-1}$.

2. We note that it is possible for a nonorientable codimension foliation of an orientable manifold to have no nonorientable leaves. To construct an example, let $\xi$ be a nonorientable line bundle over the two-dimensional torus $T^{2}$. Then $\xi \oplus \xi$ is trivial so $\xi$ can be realized as a subbundle of the tangent bundle of $T^{2}$ and therefore defines a nonorientable codimension one foliation of $T^{2}$. Since each leaf of this foliation is one dimensional, it is also orientable. It follows that, for any orientable manifold $M, M \times T^{2}$ is orientable and has a nonorientable codimension one foliation with no nonorientable leaves.

3. The argument used to prove Theorem 1 can be modified slightly to prove that the number of resilient leaves in a codimension one foliation of a paracompact manifold is countable.

1. An example. In this section, we construct a codimension one foliation of a compact orientable three-dimensional manifold $M$ with an infinite number of nonorientable leaves.

Let $X$ be a nonorientable surface of genus three. Then $\pi_{1}(X)$ has generators $\alpha, \beta, \varepsilon$ and one relation $\alpha \beta \alpha^{-1} \beta^{-1} \varepsilon^{2}=1$ (see Massey [3, p. 135], for example). Suppose $f$ and $g$ are elements of $\operatorname{Diff}\left(S^{1}\right)$ with $f$ orientation reversing, $g$ orientation preserving, and $f^{2}=$ id. We can then define a homomorphism $\varphi: \pi_{1}(X) \rightarrow \operatorname{Diff}\left(S^{1}\right)$ by setting

$$
\varphi(\alpha)=g, \quad \varphi(\beta)=\mathrm{id}, \quad \varphi(\varepsilon)=f .
$$

Let $\Gamma$ be the image of $\varphi$ in $\operatorname{Diff}\left(S^{1}\right)$. Define $M=M(f, g)$ to be the flat $S^{1}$-bundle over $X$ defined by the homomorphism $\varphi$; explicitly, $M=\tilde{X} \times S^{1} / \pi_{1}(X)$, where $\tilde{X}$ is the universal cover of $X, \pi_{1}(X)$ acts on $\tilde{X}$ by covering transformations, on $S^{1}$ using $\varphi$, and diagonally on $\tilde{X} \times S^{1}$. The leaves of the foliation $\mathcal{F}=\mathscr{F}(f, g)$ of $M$ are the images $L(z)$ of the sets $\tilde{X} \times\{z\}, z \in S^{1}$, under the natural projection $\tilde{X} \times S^{1} \rightarrow M$. The following facts are easily verified:

(1.1) $M$ is orientable.

(1.2) $L\left(z_{1}\right)=L\left(z_{2}\right)$ if and only if $z_{2}$ is in the $\Gamma$ orbit of $z_{1}$.

(1.3) $L(z)$ is nonorientable if and only if $z$ is fixed by an orientation reversing difffeomorphism in $\Gamma$.

For example, to prove (1.1), let $\bar{X} \rightarrow X$ be the two-fold orientable covering space with covering involution $h: \bar{X} \rightarrow \bar{X}$. It is not difficult to see that $\bar{X} \times S^{1}$ is a two-fold covering space of $M$ with covering involution $h \times f$. Since both $h$ and $f$ reverse orientation, $h \times f$ preserves orientation and it follows that $M$ is orientable.

Statement (1.2) follows immediately from the definition of $M$. To prove (1.3), note that a leaf $L(z)$ is nonorientable if and only if the normal bundle to $L(z)$ in $M$ is nonorientable. It is easy to see that this normal bundle is the flat line bundle over $L(z)$ defined by the linearized leaf holonomy group. Statement (1.3) now follows from the fact that the leaf holonomy group of $L(z)$ can be identified with the subgroup of $\Gamma$ fixing $z$. 
We now define explicit $f, g \in \operatorname{Diff}\left(S^{1}\right)$ with the property that the foliation $\widetilde{r}=\widetilde{G}(f, g)$ of $M=M(f, g)$ described above has an infinite number of nonorientable leaves.

Consider the circle $S^{1}$ as the real projective line $R P_{1}$, and let $f, g$ be the diffeomorphisms of $S^{1}$ given, in terms of the natural identification $S^{1}=R P_{1}=R \cup$ $\{\infty\}$ by the formulas

$$
f(x)=1 / x, \quad g(x)=x-2 .
$$

Let $\Gamma$ be the subgroup of the group of diffeomorphisms of $S^{1}$ generated by $f$ and $g$. The map $f$ is orientation reversing and of order two, while $g$ is orientation preserving. Each composite $f \circ g^{n}(n \geqslant 1)$ is orientation reversing and has fixed points $n \pm \sqrt{n^{2}+1}$. For each $n \geqslant 1$, let $x_{n}$ denote the particular fixed point $x_{n}$ denote the particular fixed point

$$
x_{n}=n+\sqrt{n^{2}+1}
$$

of $f \circ g^{n}$. In particular, each of the leaves $L\left(x_{n}\right)$ is nonorientable.

Proposition 1.1. The collection $\left\{x_{n} \mid n \geqslant 1\right\}$ of points of $S^{1}$ is not contained in a finite union of $\Gamma$-orbits.

Corollary. If $f, g \in \operatorname{Diff}\left(S^{1}\right)$ are as in (1.4), then the foliation $\mathscr{\mathcal { F }}(f, g)$ of $M(f, g)$ has an infinite number of nonorientable leaves.

The Corollary follows immediately from (1.2), (1.3) and Proposition 1.1. The proof of Proposition 1.1 is given in the next section.

2. The proof of Proposition 1.1. For each positive integer $k$, let $r(k)$ denote the collection of prime numbers which appear to an odd power in the prime factorization of $k$. The proof of Proposition 1.1 depends upon the following lemma.

LemMa 2.1. If the points $x_{n}, x_{m}$ (defined in (1.5)) lie in the same $\Gamma$-orbit, then $r\left(m^{2}+1\right)=r\left(n^{2}+1\right)$.

Proof of Proposition 1.1 (From Lemma 2.1). It is enough to show that for any integer $n \geqslant 1$ there exists an $m>n$ such that $x_{m}$ does not lie in $\Gamma\left(x_{1}\right) \cup \cdots \cup \Gamma\left(x_{n}\right)$. Let $S$ be the finite set of primes given by $S=\cup_{k=1}^{n} r\left(k^{2}+1\right)$, and let $p$ be some prime congruent to 1 modulo 4 which does not appear in $S$. (The existence of such a $p$ is guaranteed by Dirichlet's result that there are an infinite number of primes in any arithmetic progression.) The multiplicative group of nonzero residues $\bmod p$ is cyclic of order $p-1$, and, since 4 divides $p-1$, there exists a residue class $\bar{m} \bmod p$ such that $\bar{m}^{2} \equiv-1(p)$. Let $m$ be the unique positive integer less than $p$ which represents $\bar{m}$. By construction, $p$ divides $m^{2}+1$ and (since $\left.m^{2} \leqslant(p-1)^{2}\right), p^{2}$ does not divide $m^{2}+1$, so that $p \in r\left(m^{2}+1\right)$. Since $p \notin S$, it follows from Lemma 2.1 that $x_{m} \notin \Gamma\left(x_{1}\right) \cup \cdots \cup \Gamma\left(x_{n}\right)$.

Proof of Lemma 2.1. Any transformation in $\Gamma$ can be written in the form $x \mapsto(a x+b) /(c x+d)$, where $a, b, c$ and $d$ are integers. In particular, if $x_{m}$ lies in 
the $\Gamma$-orbit of $x_{n}$, there exist integers $a, b, c, d$ such that

$$
\sqrt{m^{2}+1}=(-m)+\left(\frac{a x_{n}+b}{c x_{n}+d}\right) .
$$

This implies that $\sqrt{m^{2}+1}$ lies in the quadratic extension field of $\mathbf{Q}$ generated by $\sqrt{n^{2}+1}$. Since neither $n^{2}+1$ nor $m^{2}+1$ is a perfect square, each of the fields $Q\left(\sqrt{n^{2}+1}\right)$ and $Q\left(\sqrt{m^{2}+1}\right)$ is a nontrivial quadratic extension of $Q$. It follows that $\sqrt{m^{2}+1}$ lies in $Q\left(\sqrt{n^{2}+1}\right)$ if and only if these two extensions coincide. However, if $r\left(m^{2}+1\right) \neq r\left(n^{2}+1\right)$, these extensions are distinguished from one another by the discriminant (see, for example, Marcus [2, p. 33, Exercise 1, p. 39]). This completes the proof of Lemma 2.1.

3. The proof of Theorem 1. Let $M$ be a smooth, connected, orientable, paracompact $n$-manifold, $\mathscr{F}$ a codimension one foliation of $M$ and fix a smooth, onedimensional foliation $\mathcal{E}$, transverse to $\mathscr{F}$. Note that in general, $\mathcal{E}$ is not orientable. Let $\left\{\left(U_{i}, \varphi_{i}\right) \mid i=1,2, \ldots\right\}$ be a countable, locally finite coordinate cover of $M$ which is regular with respect to both the foliations $\mathscr{F}$ and $\mathcal{L}$ on $M$. That is $\varphi_{i}: U_{i} \rightarrow D^{n-1} \times I$ are diffeomorphisms such that if $\overline{\mathcal{F}}$ and $\overline{\mathrm{E}}$ are the trivial foliations, $\left\{D^{n-1} \times\{t\} \mid t \in I\right\}$ and $\left\{\{x\} \times I \mid x \in D^{n-1}\right\}$ of $D^{n-1} \times I$ respectively, then

$$
\varphi_{i}^{-1}(\overline{\mathscr{T}})=\widetilde{\mathscr{T}}\left|U_{i}, \quad \varphi_{i}^{-1}(\overline{\mathrm{E}})=\varrho\right| U_{i}
$$

for $i=1,2$.

For each pair $(i, j)$ with $U_{i} \cap U_{j} \neq \varnothing$, fix $x_{i j} \in U_{i} \cap U_{j}$. Consider the component $J\left(x_{i j}\right)$ of the intersection of the leaf of $E$ containing $x_{i j}$ with $U_{i} \cap U_{j}$; by the choice of the $U_{i}$ 's each $J\left(x_{i j}\right)$ is a subarc of a leaf of $E$. Now $J\left(x_{i j}\right)$ can be projected along leaves in $U_{i}$ and $U_{j}$ to subarcs $J_{i}\left(x_{i j}\right)$ and $J_{j}\left(x_{i j}\right)$ of $\varphi_{i}^{-1}(\{0\} \times I)$ and $\varphi_{j}^{-1}(\{0\} \times I)$, respectively. More precisely, the projection $p_{i}: J\left(x_{i j}\right) \rightarrow J_{i}\left(x_{i j}\right)$ is defined by $p_{i}\left(\varphi_{i}^{-1}(x, t)\right)=\varphi_{i}^{-1}(0, t)$ and $p_{j}$ is defined similarly. We then obtain

$$
h(i, j)=p_{i} \circ p_{j}^{-1}: J_{j}\left(x_{i j}\right) \rightarrow J_{i}\left(x_{i j}\right),
$$

which generate the holonomy pseudogroup (see Plante [4, p. 337]). Note that for any $k$ with $U_{i} \cap U_{k} \neq \varnothing$, the composite $h(k, i) \circ h(i, j)$ maps a subarc of $\varphi_{j}^{-1}(\{0\} \times I)$ onto a subarc of $\varphi_{k}^{-1}(\{0\} \times I)$. Similarly, for any finite ordered chain $U_{i_{1}}, \ldots, U_{i_{m}}$ with $U_{i,} \cap U_{i_{j+1}} \neq \varnothing$, we have a well-defined composite $h\left(i_{m}, i_{m-1}\right) \circ \ldots \circ h\left(i_{2}, i_{1}\right)$.

Now let $\mathcal{H}$ be the set of all composites

$$
h=h\left(i_{m}, i_{m-1}\right) \circ \cdots \circ h\left(i_{2}, i_{1}\right),
$$

where $i_{m}=i_{1}, h$ is orientation reversing, and $h$ has a fixed point. We remark that since any $h \in \mathcal{H}$ is defined on a connected arc, it has exactly one fixed point which we denote by $x_{h}$. We claim that the leaf $L_{h}$ of $\mathscr{F}$ through $x_{h}$ is nonorientable. To see this choose a loop in $L_{h}$ contained in the chain $U_{i_{1}}, U_{i_{2}}, \ldots, U_{i_{m}}=U_{i_{1}}$, based at $x_{h}$. Since $M$ is oriented and $h$ reverses the orientation at $x_{h}$, this loop must also reverse the local orientation of $L_{h}$ at $x_{h}$. Thus $L_{h}$ is nonorientable.

Let $\mathfrak{X}$ be the set of all nonorientable leaves of $\mathscr{F}$ and define $\Phi: \mathcal{H} \rightarrow \mathfrak{X}$ by $\Phi(h)=L_{h}$. Now, $\Phi$ is onto because for any nonorientable $L$ any orientation reversing loop in $L$ based at $x$ can be covered by a chain $U_{i_{1}}, \ldots, U_{i_{m}}$ with 
$U_{i,} \cap U_{i_{1+1}} \neq \varnothing$ and $i_{1}=i_{m}$. Then $h=h\left(i_{m}, i_{m-1}\right) \circ \cdots \circ h\left(i_{2}, i_{1}\right)$ is orientation reversing and has $x$ as a fixed point. Since $\mathcal{H}$ is countable this completes the proof of the theorem.

\section{BIBLIOGRAPHY}

1. D. B. A. Epstein. D. C. Millett and D. Tischler, Leaves without holonomy, J. London Math. Soc. (2) 16 (1977), 548-552.

2. D. Marcus, Number fields, Universitext, Springer-Verlag, New York, 1977.

3. W. S. Massey, Algebraic topolog:: an introduction, Graduate Texts in Math., vol. 56, Springer-Verlag, New York, 1967.

4. J. F. Plante, Foliations with measure preserving holonomy, Ann. of Math. (2) 102 (1975), 327-361.

Department of Mathematics, Yale University, Box 2155, Yale Station, New Haven, ConNEC TICUT 06520 (Current address of D. B. Ellis and R. H. Szczarba)

Department of Mathematics, University of Notre Dame, Notre Dame, Indiana 46556 (Current address of W. G. Dwyer) 